\title{
Development of Composite Power System Effective Load Duration Curves by Using a New Optimization Method for Assessment Composite Generation and Transmission Reliability
}

\author{
Mohamadreza Gholami ${ }^{1}$, Reza Sharifi ${ }^{2}$, Hamid Radmanesh ${ }^{3 *}$ \\ ${ }^{1}$ Electrical Engineering Department, Zanjan University, Zanjan, Iran \\ ${ }^{2}$ Electrical Engineering Department, Amirkabir University of Technology, Tehran, Iran \\ ${ }^{3}$ Electrical Engineering Department, Shahid Sattari Aeronautical University of Science and Technology, Tehran, \\ Iran \\ *Email: hamid.radmanesh@aut.ac.ir
}

\begin{abstract}
This paper presents an approach for constructing Composite Power System Effective Load Duration Curves (CMELDC) at load points using a new optimization method based on management of an organization (MOA). The CMELDC plays an important role in subjects such as reliability evaluation and analytical outage cost assessment as it supplies some useful information. CMELDC can be obtained from convolution integral processing of the probability function of the supplied power and the load duration curve at each load point. In the proposed method, MOA intelligently searches the large state space of a power system to find the most probable states. MOA is used to represent the system states. By means of its fitness function, MOA is able to trace the probable states in the more intelligent way than the conventional methods. A linear programming model was used to check each state. After finishing the search process, CMELDC can be obtained from the states saved in the state array. To confirm the effectiveness of the proposed method in assessment of the composite system reliability indices, it is applied to Roy Billinton Test System (RBTS) and IEEE Reliability Test System (RTS) and the results are presented.
\end{abstract}

Keywords: Composite Power System Effective Load Duration Curves, Composite System Reliability Indices, Management of an Organization Based Method

\section{$1 \quad$ Introduction}

Nowadays, with a rapid growth of load demand, it is necessary for an electric utility to pay more attention to the provision of electricity for its customers in a reasonable level of reliability with economic considerations. To achieve this goal, it is necessary that the safety and reliability of providing electrical energy be measured with the proper tools. Accurate calculation reliability indices of electrical networks in expansion planning will lead to an ideal network. Also, accurate calculation and measuring of reliability indices will result in reduction of economic damage, help to find network weak points and proper operation of the network. Reliability indices calculated in the composite generation and transmission system are very complex because of the great number of equipments. On the other hand, load changing during the day and different seasons add the complexity of computations, especially with the aim of identifying weak points or proper expansion if reliability indices are required in the buses instead of generic. Because of these reasons, achieving proper tools for reliability indices calculation is a problem. A power system including generation, transmission and distribution system CMELDC can be applied usefully to subjects such as reliability evaluation, probabilistic production cost simulation, and analytical outage cost assessment [1]. Generation and composite power system reliability indices can be calculated by ELDC and CMELDC. ELDC has been used widely since Baleriaux and Jamoulle in 1967 [2] developed the recursive equations which considered the forced outage rate of generators within the LDC to get probabilistic production costs and the reliability indices. The effective load duration curve, tentatively CMELDC, based on effective load model of composite power system has been already proposed by Jaeseok Choi [1], [3] and [4]. Also, Jaeseok Choi proposed a fuzzy ELDC model for 
reliability evaluation of transmission system using fuzzy set theory [5]. Some analytical and simulationbased methods are available, but more realistic, simpler and easier to apply techniques to get CMELDC studies can be still useful.

Intelligent optimization methods, such as genetic algorithm (GA) and particle swarm optimization (PSO), have shown a rapid growth of applications in power systems [6-8]. Application of intelligent optimization methods to power systems is found in areas such as economic dispatch, power system planning and the load flow problems.

In many articles, various algorithms to combine benefits of these ways, in the form of making combined optimization are presented. In this paper, a new method of making optimal based management of an organization (MOA) is presented. In these applications, optimization algorithms are used primarily as an optimization tool. This paper uses MOA as a smart state sampling tool.

The rest of this paper has been organized as follows:

Section 2 provides a reliability evaluation of composite electric power systems. Section 3 introduces the proposed method. Section 4 explains the algorithmic structure. Simulation results together with model validation under different test systems are presented in Section 5. Finally, Section 6 concludes the paper by summarizing the results.

\section{$2 \quad$ Reliability Evaluation of Composite Electric Power Systems}

The following section is a brief introduction of composite power system reliability. Two reliability indices (LOLE and EENS) are described. Assessment methods in the power systems are mainly applied to three different hierarchical levels. At the hierarchical level I (HLI), the total system generation is examined to determine its adequacy to meet the total system load requirements. The transmission system and its ability to transfer the generated energy to the consumer load points is ignored in HL(I). In HL(II) studies, the adequacy analysis is usually termed composite system or a bulk transmission system evaluation. HLI(II) includes three main components, namely generation, transmission and distribution to consumers. Considering the complexity of systems and plants, transmission lines and distribution equipment, reliability and index calculation at this level is difficult and complex. Furthermore, the generation and transmission systems are not considered. These segments are shown in Fig. (1) [11].

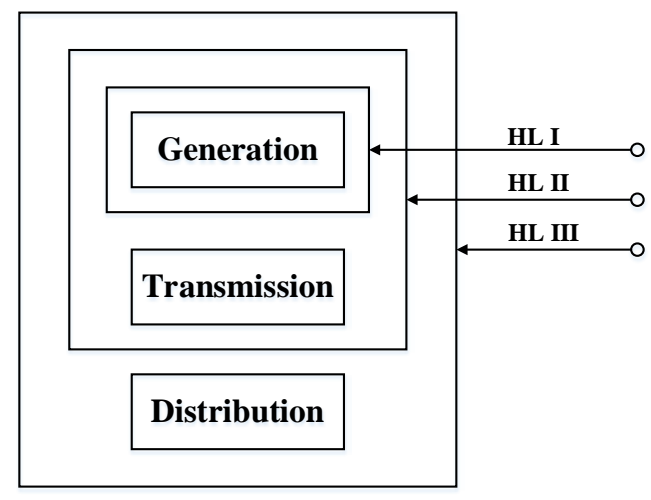

Figure 1. Power system Hierarchical Levels (HL)

Adequacy assessment of composite generation-transmission systems is a more complex task. It is divided into two main parts, i.e., state sampling and state evaluation. Each sampled state consists of the states of generating units and transmission lines, some of them are in upstate and others are in down state.

Adequacy indices for the system and for each load bus are calculated using CMELDC. These indices include Loss of Load Expectation (LOLE) and Expected Energy Not Supplied (EENS). Adequacy indices for the each load bus \#i are calculated using (1) and (2): 


$$
\begin{aligned}
\text { EENS }_{i} & =\int_{A P_{i}}^{A P_{i}+P D_{i}} C M E L D C_{i} d x \\
\text { OOLP }_{i} & =\left.C M E L D C_{i}\right|_{x}=A P_{i}
\end{aligned}
$$

then, the adequacy indices for the whole system are calculated as follows:

$$
\begin{aligned}
& \operatorname{EENS}_{H L I I}=\sum_{i=1}^{N L} E^{2} E N S_{i} \\
& L O L E_{H L I I}=\sum_{i=1}^{N L} L O L E_{i}
\end{aligned}
$$

where, $N L$ is the total number of system's load points.

\section{$3 \quad$ Introduction of a Proposed Method}

In this study, CMELDC is obtained using MOA that searches the state space to scan the most probable states. MOA is used for sampling the composite power system network states. Population of MOA is composed of persons, each person representing a network state, i.e., the states of generating units and transmission lines. MOA search process is guided through its fitness function. States sampled are saved with their data in the state array. After finishing the search process, states saved in the state array are evaluated by a linear programming model and are used to get the CMELDC. It is expected that the CMELDC proposed in this study will be useful for above applications of CMELDC in electric utility planning and power system reliability studies of complex systems.

Other methods, in this way, first generation randomly is created and then changes are done on the generation in various iterations, until the end condition is established. Difference of this method with methods such as genetic algorithms and PSO algorithm that it is not inspired by natural factors, but based on successful performance of an organization.

In order to proper function of an organization, all staff should be associated with their deputies and act through the general goals of the organization determined by the chief. While in a successful organization, most valuable people are in better positions and creativity and personal development in the people can be seen.

Scheme of this algorithm is that after producing the first population, based on fit people, the best person as president and the next three as deputy chairman are elected. Other members are placed randomly in each sub-deputy.

Then the deputies move to the president. The same motion of particles moving in the PSO algorithm, toward the best particles experience, through the following formula, that is:

$$
\begin{gathered}
v_{i}(t)=v_{i}(t-1)+\rho_{1}\left(x_{\text {boss }}-x_{i(t)}\right) \\
x_{i}(t)=x_{i}(t-1)+v_{i}(t)
\end{gathered}
$$

where, $x_{\mathrm{i}}(t)$ is the position of person $i$ and $x_{\mathrm{boss}}$ is the position of the best person named boss. Each people exchanges the information with his deputies. This operator is like the coupled operation (Cross Over) in genetic algorithms. Fitted value for the generation of the above changes is re-calculated again and the best person is elected as chairman and three dimensional as his deputies. This action continues until the end of the condition. The system parameters such as initial velocity $(v)$, the factor $p_{1}$, the population size and the cross over type have been determined based on the quality of convergence. Other parameters control the value of the convergence. Small value of the parameters causes to receive the answer quickly but the possibility of mistake increases as well. This flowchart is given in Fig. 2.

\section{$4 \quad$ Algorithmic Structure}

The flowchart of this method is shown in Fig. 3. In this method, each generating unit and transmission line has its own Forced Outage Rate (FOR). The probability of any unit or line down is equal to its FOR. The total number of states for all possible combinations of $n$ generating units and transmission lines in the system is $K=2 n$. 


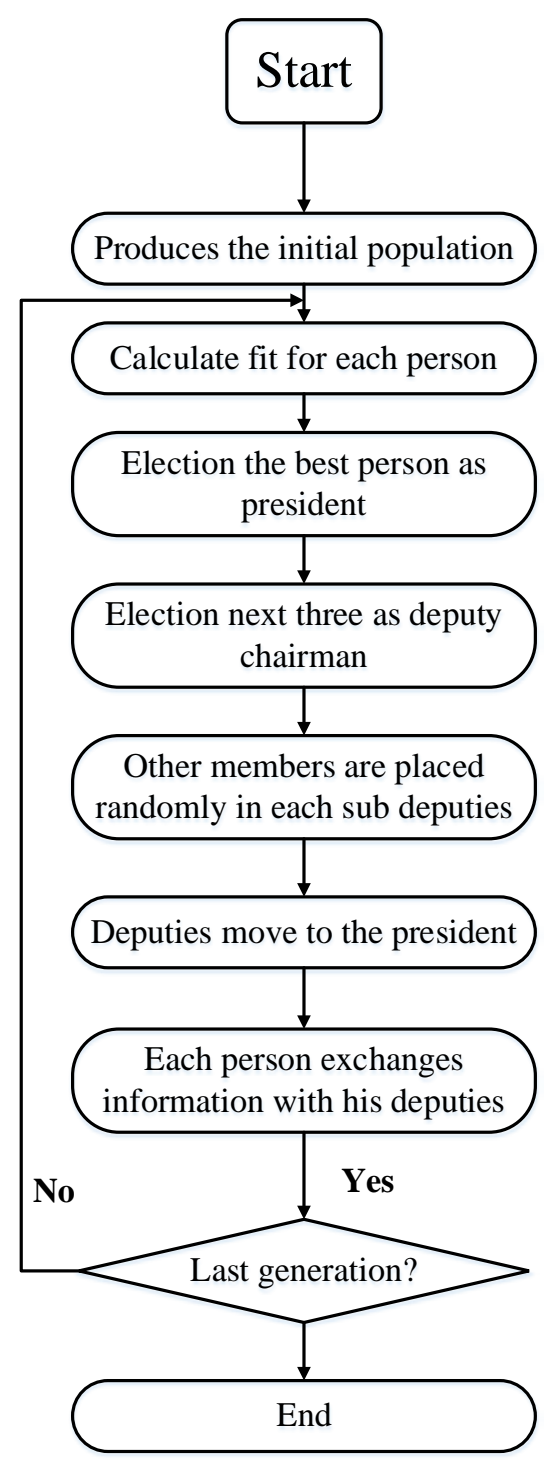

Figure 2. Flow chart of MOA

\subsection{Evaluating the Normal System State}

The state evaluation model is based on DC load flow equations. In the normal state, all units and transmission lines are in upstate. From this state maximum arrival power at each bus is calculated. The linear programming maximization problem is formulated as follows:

$$
\max \sum_{i=1}^{n l} \alpha_{i} P D_{i} \quad i=1,2,3, \ldots \ldots, n
$$

This aim is subjected to the following constraints, expressed by equations (2) - (6).

$i$. Active power balance at each system bus.

ii. Active power flow limits at each transmission line.

iii. Maximum amount of load curtailed at each load bus.

$i v$. Maximum and least available active power at each PV bus.

$$
P G_{i}-\sum_{j=1}^{k} P g_{j}-\alpha_{i} P D_{i}=0 \quad k=1,2,3, \ldots \ldots, n
$$




$$
\begin{gathered}
P G_{i \min } \leq P G_{i} \leq P G_{i \max } \quad k=1,2,3, \ldots \ldots, n_{v} \\
p l_{i \min } \leq p l_{i} \leq p l_{i \max } \quad k=1,2,3, \ldots \ldots, n_{t} \\
p_{j i}=\left(\theta_{i}-\theta_{j}\right) / X_{i j} \quad k=1,2,3, \ldots \ldots, n \\
A P_{i}=\alpha_{i} P D_{i} \quad k=1,2,3, \ldots \ldots, n
\end{gathered}
$$

where,

$n$ : a total number of system buses

$n_{\mathrm{t}}$ : a total number of the transmission lines

$n_{1}$ : a total number of load buses

$n_{\mathrm{v}}$ : a total number of buses which have generating unit

$\theta_{i}$ : the voltage angle at bus $i$

$P D_{\mathrm{i}}$ : a load demand at bus $i$ in the peak load condition,

$P G_{\mathrm{i}}$ : an active power generation at bus $i$,

$P G_{\text {imax }}$ : the maximum available active power generation at bus $i$,

$P G_{\text {imin }}$ : the minimum available generation at bus i

$P_{\mathrm{ijj}}$ : the power flowing from bus $i$ to $j$

$p l_{i}$ : the power transmission at line $i$

$p l_{\text {max }}$ : the maximum power transmission at line $i$

$p l_{\text {imin }}$ : the minimum power transmission at line $i$

$A P_{\mathrm{i}}$ : the maximum arrival power at bus $i$

\subsection{Generating the Initial Population}

In this state, an initial population is randomly created equal to the number of chromosomes "pop size". Each state " $i$ " has its own probability " $P S_{\mathrm{i}}$ ". The state probability " $P \mathrm{~S}_{\mathrm{i}}$ " for each chromosome " $i$ " is calculated.

$$
P S_{i}=\prod_{i=1}^{n} f_{i}
$$

where, $f_{\mathrm{i}}=1-F O R_{\mathrm{i}}$ if its gene $=1$ (up state) or $f_{\mathrm{i}}=F O R_{\mathrm{i}}$ if its gene $=0$ (down state).

\subsection{Evaluating the New States}

If the state probability calculated in (11) is bigger than the threshold value $\left(P_{\mathrm{th}}\right)$, the linear programming is recalled. The equations of linear programming are the same as equations (6-10) and only equation 6 is changed to:

$$
A P_{i}^{c}=\alpha_{i}^{c} P D_{i}
$$

where:

$A P_{\mathrm{i}}^{\mathrm{c}}$ : is the maximum arrival power at bus $i$ in the new state. Then this state with its data (probability state and $\left(A P_{\mathrm{i}}-A P_{\mathrm{i}}^{\mathrm{c}}\right)$ are saved in a matrix (save matrix).

$\alpha_{i}^{c}$ : represents the relation between maximum power of bus-bar $i$ and maximum electrical load demand of that bus-bar.

However, in each test case $P_{\text {th }}$ can be changed based on the electrical network configuration. Because of reference [14] which has been cited in the revised version of the paper, $P_{\mathrm{th}}$ has been considered as a parameter for RBTS network and its value is $\mathrm{e}^{-10}$ and for RTS-96 its value is $\mathrm{e}^{-20}$.

\subsection{Reproducing a New Population Using GA}

The fitness of each person " $i$ " is calculated by:

$$
\text { fitt }=P S_{i}
$$

Then MOA operators are applied to reproduce a new population. 


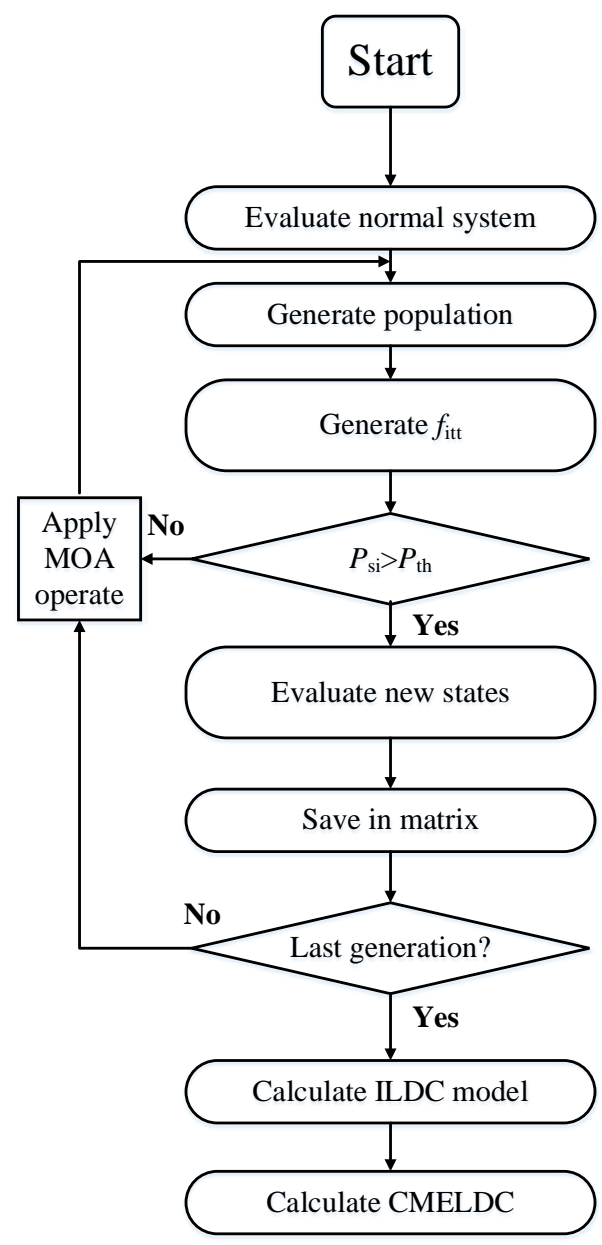

Figure 3. The flowchart of the proposed method

\subsection{Comparing With the Stopping Criterion}

The stopping criterion is to stop after reaching a certain amount MOA generations, or at the scanning of a certain number of system states by adding all numbers of the permutations in the save matrix. The second criterion gives more accurate results.

\subsection{Calculating the ILDC Model for Each Load Point}

In this study ILDC is obtained using $V_{\mathrm{PI}}$ model [12]. This model is only related to the peak load, base load and the energy. The model has been presented in [12]. For more details about $V_{\mathrm{PI}}$ model see reference [5].

\subsection{Obtaining the CMELDC}

After that the MOA stops the search process, CMELDC is calculated using saved states in the save matrix. The equation of CMELDC at load point $\# k$ is expressed by (16).

$$
\begin{aligned}
& C M E L D C_{K}=p_{1} p_{2} p_{3} f\left(A P_{k}-A P_{k i}^{C}\right)+p_{1} p_{2} q_{3} f\left(A P_{k}-A P_{k i}^{C}\right)+p_{1} q_{2} p_{3} f\left(A P_{k}-A P_{k i}^{C}\right)+ \\
& q_{1} q_{2} p_{3} f\left(A P_{k}-A P_{k i}^{C}\right)+q_{1} p_{2} q_{3} f\left(A P_{k}-A P_{k i}^{C}\right)+p_{1} q_{2} q_{3} f\left(A P_{k}-A P_{k i}^{C}\right)+q_{1} q_{2} q_{3} f\left(A P_{k}-A P_{k i}^{C}\right)
\end{aligned}
$$

For conciseness, the analytical expression for ELDC is derived only for three units. 
where, $A P_{\mathrm{k}}$ is the maximum arrival power at load point $\# k$, and $A P_{\mathrm{Ki}}{ }^{\mathrm{C}}$ is the maximum arrival power at load point \#k in sampled state $i$.

CMELDC curve is a LDC convolution and it is error rate of electrical Plants. Using ILDC curve results in the following equation:

$$
\begin{aligned}
& E L D C=p_{1} p_{2} p_{3} f\left(x_{i}\right)+p_{1} p_{2} q_{3} f\left(x_{i}-M W_{3}\right)+p_{1} q_{2} p_{3} f\left(x_{i}-M W_{2}\right)+q_{1} p_{2} p_{3} f\left(x_{i}-M W_{1}\right)+ \\
& q_{1} q_{2} p_{3} f\left(x_{i}-M W_{1}-M W_{2}\right)+q_{1} p_{2} q_{3} f\left(x_{i}-M W_{1}-M W_{3}\right)+p_{1} q_{2} q_{3} f\left(x_{i}-M W_{2}-M W_{3}\right)+\ldots \ldots
\end{aligned}
$$

where, $p_{\mathrm{i}}$ is the possibility that unit $i$ is in service, $q_{\mathrm{i}}$ is the possibility that unit $i$ is not in service and $f(\mathrm{x})$ is the model of ILDC for load $x$.

$$
\begin{gathered}
f(x)= \begin{cases}1 & \mathrm{x} \leq \mathrm{B} \\
(1-(X-B) /(P-B)) \exp \{\lambda\} & \mathrm{B} \leq \mathrm{x} \leq \mathrm{P} \\
0 & \mathrm{x} \geq \mathrm{P}\end{cases} \\
\lambda=((X-B) /(P-B)) \sum_{n=1}^{7} A_{n}\left[\frac{E-h \cdot B}{h \cdot(P-B)}-.05\right]^{n}
\end{gathered}
$$

The equation (19) shows that ELDC is considered for three units but for $\mathrm{n}$ power unit $2 n$ condition should be calculated. The higher degree of calculation increases the time of simulation and it is not acceptable. Furthermore, the Genetic algorithm is used to analyze the system behavior. This algorithm calculates the largest injection power to the load $\left(A P_{\mathrm{i}}\right)$ and saves data into the matrix. The values of the matrix find the CMELDC for next level. Finally, the calculation of reliability indices is done using CMELDC curve. This is a routine work because EENS indices can be obtained using integral of CMELDC curve from the largest power injected to the load $A P_{\mathrm{i}}$ till the largest power injected to the load at bus-bar $I\left(A P_{\mathrm{i}}+\right.$ Maxload $\left._{\mathrm{i}}\right)$. In addition, $L O L E_{\mathrm{i}}$ for $A P_{\mathrm{i}}$ is calculated. Fig. 4 shows the $E E N S$ and $L O L E$ calculation method.

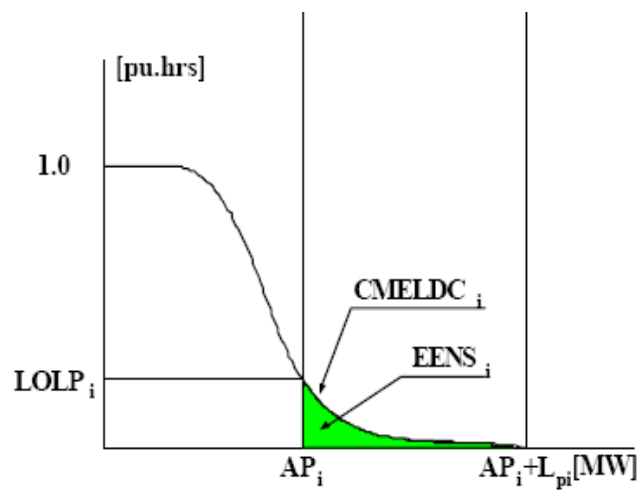

Figure 4. Scheme of EENS and LOLE calculation using CMELDC curve

\section{$5 \quad$ Case Study}

The proposed method has been tested on the RBTS [13] and the larger RTS-96 system [9].

\subsection{Case I: RBTS}

At first, the proposed method has been tested on the RBTS system as a small test system. Single line diagram of the RBTS system is shown in Fig. 5. It consists of 2 generators $(P V)$ buses, 4 loads $(P Q)$ buses, 9 transmission lines and 11 generating units. Considering the normal state in which all the system components are up, the person representing this state is shown in Table 1. 


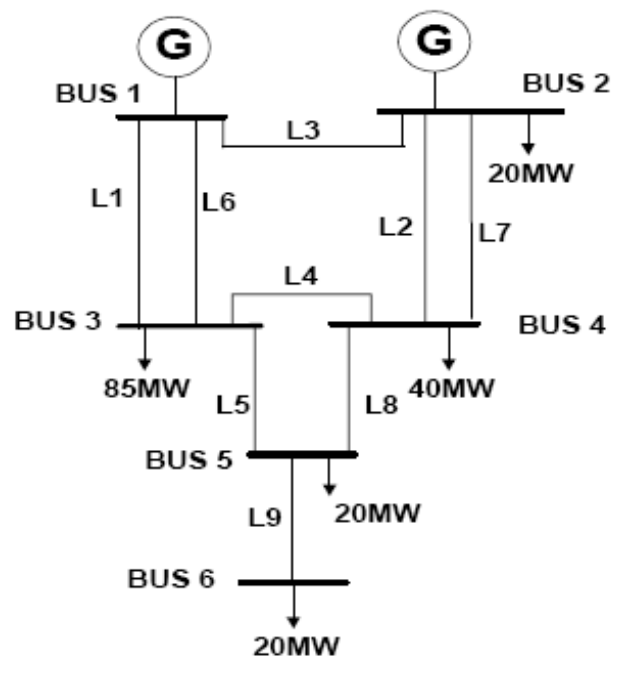

Figure 4. Single line diagram of the RBTS

The used stopping criterion is the total number of MOA generations which is selected to be 2000. The CMELDC obtained for some load points in this case study are shown in Figs. 6-8. Adequacy indices for each load point, calculated by the proposed MOA based method, are given in Table 2 . In Table 3 , the reliability for system is compared with the original values in different generations of MOA.

In Table 4, the results for MOA 2000 iteration have been compared with the results of reference [14].

Table 1. Person representing the normal state

\begin{tabular}{cccc}
\hline Generation Units & States* & Transmission Lines & States** \\
\hline $40 \mathrm{MW}$ & 1 & L1 & 1 \\
$40 \mathrm{MW}$ & 1 & L2 & 1 \\
$20 \mathrm{MW}$ & 1 & L3 & 1 \\
$10 \mathrm{MW}$ & 1 & L4 & 1 \\
$40 \mathrm{MW}$ & 1 & L5 & 1 \\
$20 \mathrm{MW}$ & 1 & L6 & 1 \\
$20 \mathrm{MW}$ & 1 & L7 & 1 \\
$20 \mathrm{MW}$ & 1 & L8 & 1 \\
$20 \mathrm{MW}$ & 1 & L9 & 1 \\
$5 \mathrm{MW}$ & 1 & - & - \\
$5 \mathrm{MW}$ & 1 & - & - \\
\hline * Generation Units or Transmission Lines: States $=0$ (down state) \\
** Generation Units or Transmission Lines: States =1 (up state)
\end{tabular}

Table 2. Adequacy indices for RBTS calculated by the proposed MOA based method

\begin{tabular}{ccc}
\hline $\begin{array}{c}\text { BUS } \\
\text { Number }\end{array}$ & $\begin{array}{c}\text { LOLE } \\
\text { hr/yr }\end{array}$ & $\begin{array}{c}\text { EENS } \\
\text { MWh }\end{array}$ \\
\hline BUS\#2 & 1.73 & 3.79 \\
BUS\#3 & 0.0785 & 33.4913 \\
BUS\#4 & 6.66 & 31.21 \\
BUS\#5 & 2.76 & 7.61 \\
BUS\#6 & 11.44 & 129.4874 \\
\hline
\end{tabular}




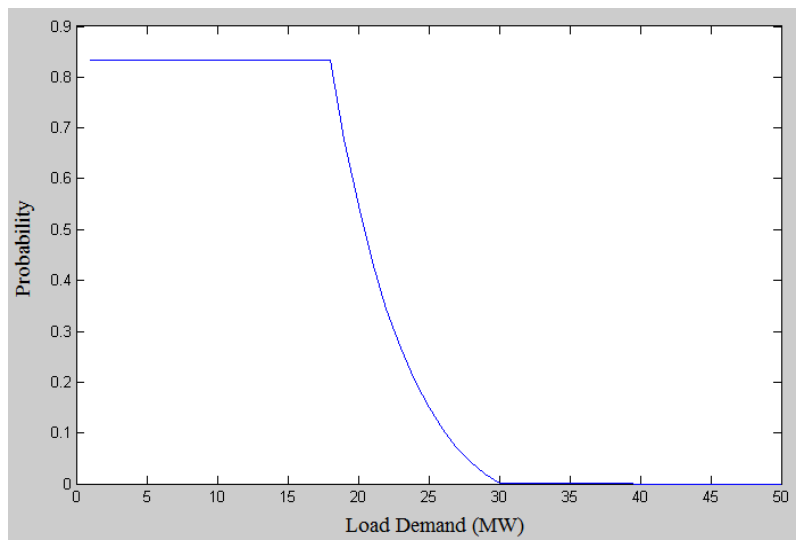

Figure 5. CMELDC for load point \#2(RBTS)

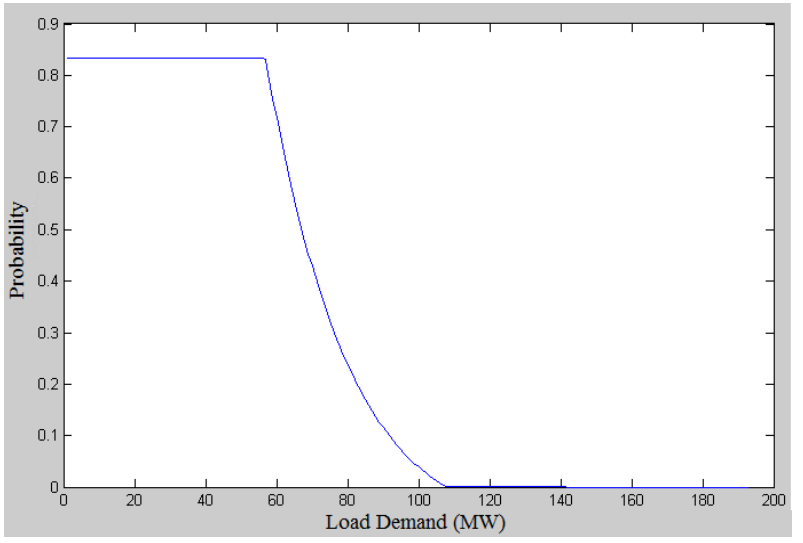

Figure 6. CMELDC for load point \#3(RBTS)

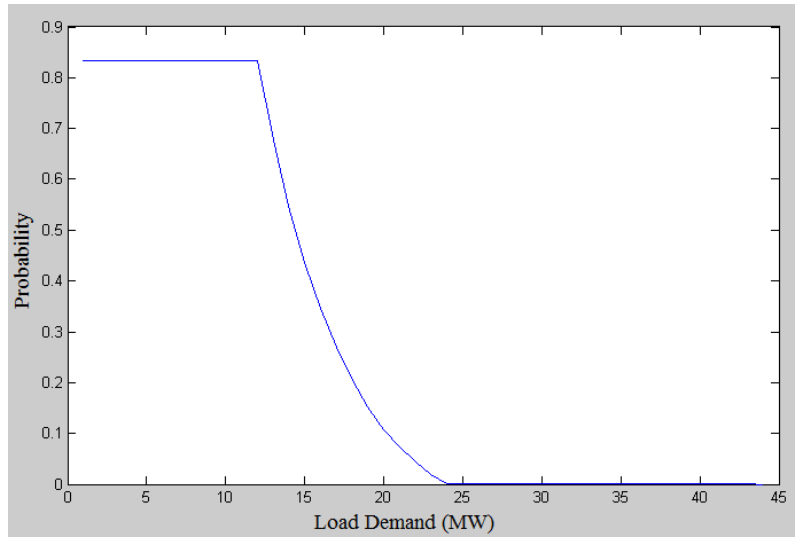

Figure 7. CMELDC for load point \#6(RBTS)

The results show that bus-bar 6 has maximum power losses and maximum blackout hours to be allocated to this bus-bar. 
Table 3. Comparison of indices in different generations of MOA

\begin{tabular}{l|l|l|l|l|l}
\hline \multirow{2}{*}{ Index } & \multicolumn{5}{|c}{ Method: MOA, Iteration } \\
\cline { 2 - 6 } & 700 & 1000 & 1300 & 2000 & Sequential sampling [10] \\
\hline LOLE hr/yr & 80.42 & 84.92 & 86.01 & 86.181 & 86.399 \\
\hline EENS Wh & 998.1 & 1067.1 & 1080.8 & 1080.92 & 1081.01 \\
\hline
\end{tabular}

Table 4. Comparison of MOA 2000 iteration with reference [14]

\begin{tabular}{c|c|c}
\hline Reliability Indices & MOA Algorithm & GA Algorithm [14] \\
\hline LOLE hr/yr & 86.181 & 85.20 \\
\hline EENS MWh & 1080.92 & 1047.78 \\
\hline
\end{tabular}

\subsection{Case II: RTS-96}

To verify the accuracy of the proposed method in large systems, the proposed method was also tested on RTS-96, as a large system. Single line diagram of the RTS-96 test system is shown in Fig. 9. It consists of 10 generators (PV) buses, 38 transmission lines and 32 generating units. The total installed capacity is $3405 \mathrm{MW}$ with a peak load of $2850 \mathrm{MW}$. Each person which represents a network state, consists of $(32+38)$ gene, where 32 is the number of generating units and 38 is the number of transmission lines. For the RTS 96 network, CMELDC curves for bus-bar 2, 3 and 4 have been shown in Figs. 10, 11 and 12, respectively. The values of indices LOLE and EENS are related to loads and they have been obtained using curve.

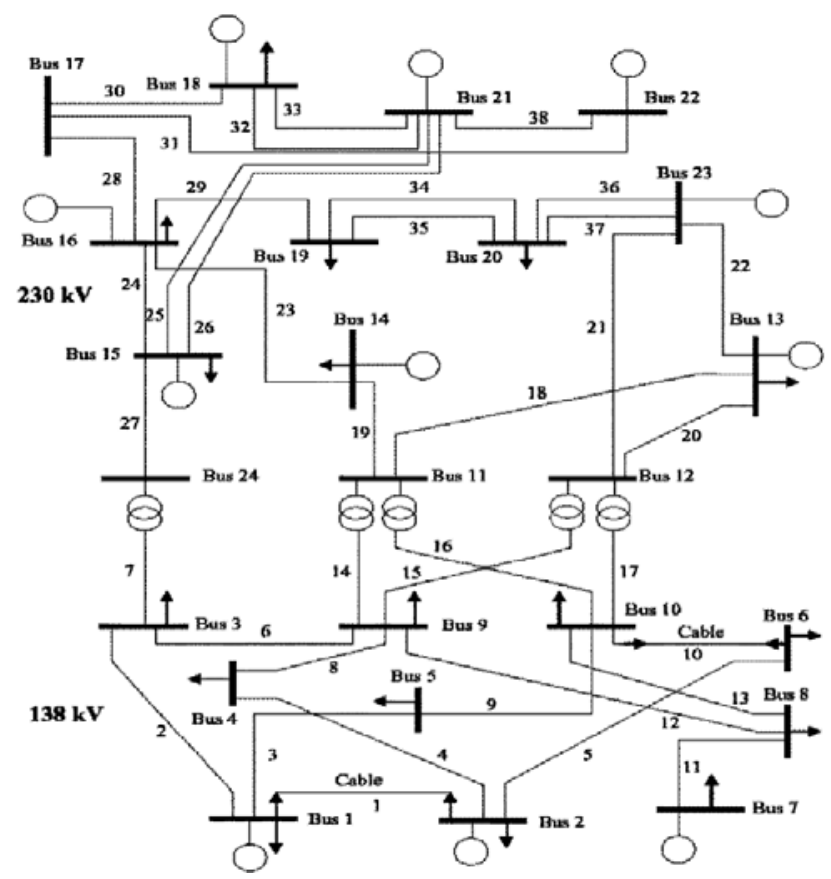

Figure 8. Single line diagram of the RTS 


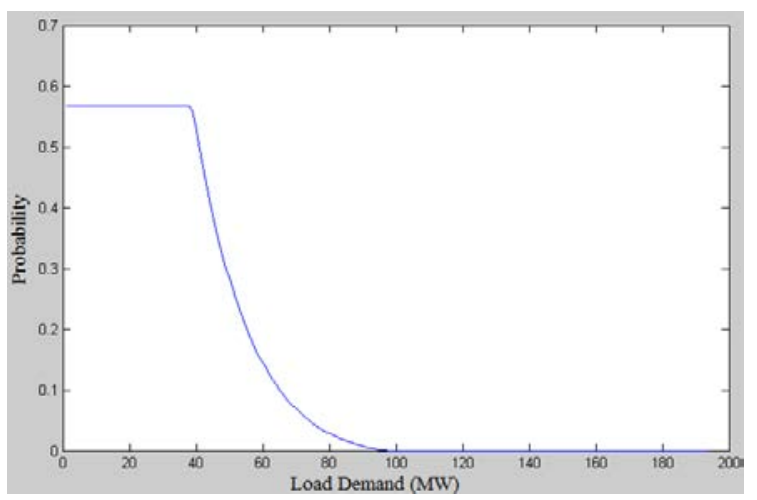

Figure 9. CMELDC for load point \#2(RTS-96)

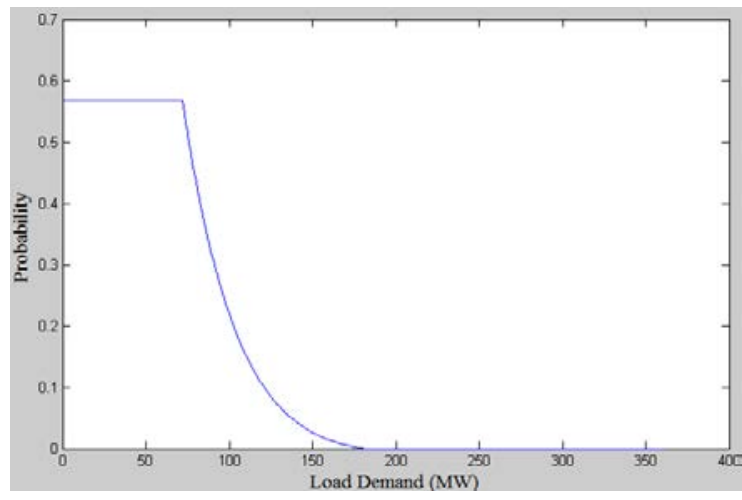

Figure 10. CMELDC for load point \#3(RTS-96)

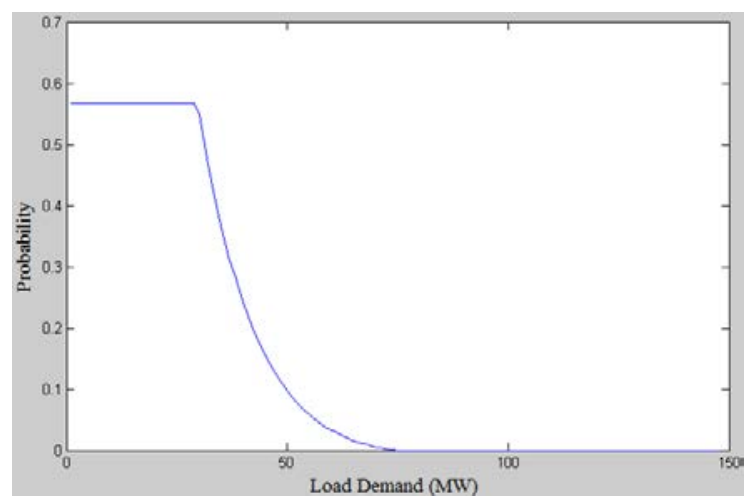

Figure 11. CMELDC for load point \#4(RTS-96)

In Table 5 the system reliability indices of the RTS-96, which have been determined by the proposed method, are compared with the real values of indices. The results show that the indices can be calculated from CMELDC by using MOA in an efficient way and less computation. 
Table 5. Adequacy indices for RTS calculated by the proposed MOA based method

\begin{tabular}{c|c|c}
\hline System Indices & Original & MOA \\
\hline LOLP hr $/$ yr & .000998 & .00098 \\
\hline EENS MWh & 1095 & 1093 \\
\hline
\end{tabular}

\section{Conclusion}

CMELDC plays an important role in subjects such as reliability evaluation and analytical outage cost assessment as it supplies some very useful information. This paper presents an approach based on an optimization method (MOA), as a smart state sampling tool, to construct composite power system effective load duration curves. MOA intelligently searches the large state space of a power system to find the most probable states. A linear programming model has been used to check each state. Then, CMELDC can be obtained from sampled states. The proposed method has been applied on the RBTS and the larger RTS-96 system and the results are presented. The simulation results show that the proposed method is more effective than the conventional methods when the system becomes larger.

\section{References}

1. Jaeseok Choi, Seungpil Moon, Hongsik Kim, Byongjun Lee and R. Billinton, "Development of the ELDC and reliability evaluation of composite power system using Monte Carlo method," Power Engineering Society Summer Meeting, 2000. IEEE, Seattle, WA, vol. 4, 2000, pp. 2063-2068.

2. A. J. Wood and B.F. Wollenberg, "Power Generation Operation and Control," John Wiley EJ Sons, 1984.

3. Jeaseok Choi, "Outage Cost Assessment using Analytical Method on Composite Power System," Final Report of Post-Doctor Course at University of Saskatchewan, February 1997.

4. Jaeseok Choi, Daeho Do, Seungpil Moon and Roy Billinton, "Development of a Method for ELDC Construction in a Composite Power System," Proceedings of Large Engineering Systems Conference on Power System, Halifax, NS, Canada, June 20-22 1999, pp.189-194.

5. Jaeseok Choi, Hongsik Kim, Seungpil Moon, Junmin Cha, Daeseok Rho and R. Billinton, "A study on the fuzzy ELDC of composite power system based on probabilistic and fuzzy set theories," Power Engineering Society Summer Meeting, 2002 IEEE, Chicago, IL, USA, vol.3, 2002, pp. 1123-1129.

6. Ganjehkaviri, A., et al., "Genetic algorithm for optimization of energy systems: Solution uniqueness, accuracy, Pareto convergence and dimension reduction," Energy, 119, pp.167-177,2017.

7. Tezer T, Yaman R, Yaman G., "Evaluation of approaches used for optimization of stand-alone hybrid renewable energy systems," Renewable and Sustainable Energy Reviews. vol.73, pp.40-53, 2017.

8. Shahgholian, Ghazanfar, et al., "Power system oscillations damping by optimal coordinated design between PSS and STATCOM using PSO and ABC algorithms," 2016 13th International Conference on Electrical Engineering/Electronics, Computer, Telecommunications and Information Technology (ECTI-CON),. IEEE, 2016.

9. IEEE RTS Task Force of APM Subcommittee, "IEEE reliability test system," IEEE PAS, vol. 98, no. 6, pp. 2047-2054, 1979.

10. R. Billinton and A. Sankarakrishnan, "A comparison of Monte Carlo simulation techniques for composite power system reliability assessment," WESCANEX 95. Communications, Power, and Computing. Conference Proceedings, IEEE, Winnipeg, Man., vol.1, 1995, pp. 145-150.

11. R. Billinton and L. Gan, "Use OF Monte Carlo Simulation IN Teaching Generating Capacity Adequacy Assessment," IEEE Transactions on Power Systems, Vol. 6, No. 4, pp.1571-1577, 1991.

12. S. Rahman, Rinaldy, "An Efficient Load Model for Analyzing Demand Side Management Impacts," IEEE Transactions on Power Systems, Vol. 8, No. 3, 1993.

13. R. Billinton et al., "A reliability test system for educational purposes-basic data," IEEE Transactions on Power Systems, vol. 4, no. 3, pp. 1238-1244, 1989.

14. Nader Samaan, Phd Thesis, Texas A\&M University, "Reliability Assessment of Electric Power Systems Using Genetic Algorithms," August 2014. 